\title{
Myocardial involvement and deformation abnormalities in idiopathic inflammatory myopathy assessed by CMR feature tracking
}

\author{
Johannes Kersten ${ }^{1,6}$ (1) Ahmet Muhammed Güleroglu ${ }^{2} \cdot$ Angela Rosenbohm $^{3} \cdot$ Dominik Buckert $^{1}$. \\ Albert Christian Ludolph ${ }^{3}$. Carsten Hackenbroch ${ }^{4} \cdot$ Meinrad Beer $^{5} \cdot$ Peter Bernhardt ${ }^{2}$
}

Received: 15 April 2020 / Accepted: 4 September 2020 / Published online: 17 September 2020

(c) The Author(s) 2020

\begin{abstract}
Background Cardiac involvement has been described in idiopathic inflammatory myopathies (IIM), including non-specific ECG and echocardiographic findings. Aim of our study was to evaluate myocardial deformation parameters in IIM and to correlate them with late gadolinium enhancement (LGE) findings using cardiac magnetic resonance imaging (CMR).

Methods Forty-seven consecutive patients with histologically proven IIM were included into our study. Twenty-five healthy volunteers were used as a control group. All patients and controls underwent CMR examination using a $1.5 \mathrm{~T}$ scanner including functional cine and LGE imaging. After a mean follow-up of $234.7 \pm 79.5$ days a second CMR examination was performed in IIM patients.

Results In comparison to healthy volunteers, IIM patients had lower left ventricular mass and left ventricular global radial, circumferential and longitudinal strain. There was no significant difference in left ventricular ejection fraction.

Patients with LGE $(\mathrm{N}=28)$ had lower left ventricular ejection fraction $(\mathrm{p}=0.016)$, global right and left ventricular longitudinal strain $(p=0.014$ and $p=0.005)$ and global left ventricular diastolic longitudinal strain rate $(p=0.001)$ compared to patients without LGE $(\mathrm{N}=19)$.

In IIM patients, a significant decrease of left ventricular ejection fraction, left ventricular mass and all measured deformation parameters was observed between baseline and follow-up CMR.

Conclusion Cardiac involvement in IIM is frequent. Impairment of systolic and diastolic deformation parameters and a worsening over time can be observed. CMR is a useful tool for cardiac diagnostic work-up of these patients.
\end{abstract}

Keywords Idiopathic inflammatory myopathies $\cdot$ Cardiac magnetic resonance $\cdot$ Late gadolinium enhancement $\cdot$ Strain imaging

Johannes Kersten

johannes.kersten@uni-ulm.de

Ahmet Muhammed Güleroglu

muhammed.gueleroglu@uni-ulm.de

Angela Rosenbohm

angela.rosenbohm@uni-ulm.de

Dominik Buckert

dominik.buckert@uni-ulm.de

Albert Christian Ludolph

albert.ludolph@rku.de

Carsten Hackenbroch

carstenhackenbroch@bundeswehr.org

Meinrad Beer

meinrad.beer@uniklinik-ulm.de

Peter Bernhardt

peter.bernhardt@herzklinik-ulm.de
1 Department of Internal Medicine II, University of Ulm, Albert-Einstein-Allee 23, 89081 Ulm, Germany

2 Heart Clinic Ulm, Magirusstr. 49, 89077 Ulm, Germany

3 Department of Neurology, University of Ulm, Oberer Eselsberg 45, 89081 Ulm, Germany

4 Department of Radiology, Armed Forces Military Hospital Ulm, Oberer Eselsberg 40, 89081 Ulm, Germany

5 Department of Diagnostic and Interventional Radiology, University of Ulm, Albert-Einstein-Allee 23, 89081 Ulm, Germany

6 University Hospital of Ulm, Albert-Einstein-Allee 23, Ulm, Germany 


\section{Abbreviations \\ CMR Cardiovascular magnetic resonance \\ IIM Idiopathic inflammatory myopathy \\ LGE Late gadolinium enhancement \\ LV Left ventricular \\ LVEDVi Left ventricular end-diastolic volume index \\ LVEF Left ventricular ejection fraction \\ LVGCS Left ventricular global circumferential strain \\ LVGLS Left ventricular global longitudinal strain \\ LVGRS Left ventricular global radial strain \\ RVEDVi Right ventricular end-diastolic volume index \\ RVEF Right ventricular ejection fraction \\ RVGLS Right ventricular global longitudinal strain}

\section{Introduction}

Idiopathic inflammatory myopathies (IIM) are potentially treatable chronic autoimmune disorders with muscle weakness, muscle enzyme elevation, and extra-muscular manifestations [1,2]. They include different well-characterized disorders including dermatomyositis, polymyositis, necrotizing autoimmune myositis, inclusion-body myositis and overlap syndrome [2]. Cardiac involvement is a common finding in these patients and is reported in about 32 to $77 \%$ [3]. In comparison to the general population, patients with IIM have an increased risk for cardiac death [4]. Thereby, an early detected cardiac involvement of IIM could have influence on therapeutic regime leading to improvement of survival [5]. Accordingly, the Working Group on Myocardial and Pericardial Diseases of the European Society of Cardiology detected a lack in diagnostic work-up for patients with inflammatory diseases [6]. Classical physical stress tests and laboratory tests are prone to error in IIM patients because of muscular fatigue and muscular isoenzyme interactions [7].

Cardiac magnetic resonance (CMR) imaging has become the standard non-invasive imaging modality in inflammatory myocarditis [8] and for detection of cardiac involvement in systemic muscle diseases [9-11]. It has also been shown to be a valuable tool for detection of cardiac involvement in patients with IIM $[10,11]$. With modern methods of feature tracking, measurement of myocardial deformation has shown its prognostic value in different cardiac entities [12, 13]. Aim of our study was to evaluate myocardial deformation parameters in IIM and their development over time. Deformation parameters were correlated with Late gadolinium enhancement (LGE) findings and compared with an age and gender matched control group using cardiac magnetic resonance imaging.

\section{Materials and methods}

\section{Study population}

Patients with known and treated IIM and patients presenting with suspected inflammatory myopathy were screened for inclusion into the study. Before enrollment the diagnosis had to be histopathologically proven by skeletal muscle biopsy. CMR-Scans were performed at baseline and after a follow-up of about 6 to 9 months. A control group of healthy volunteers were screened for age and gender match.

Exclusion criteria were contraindication for CMR, gadolinium-based contrast agent, pregnancy, unknown contraception or a severely reduced condition due to immobility because of severe paresis or the diagnose of inclusion body myositis. All included patients gave informed written consent. The study was approved by the local ethics committee (approval no. 13/10).

\section{Cardiac magnetic resonance}

\section{Image acquisition}

CMR was performed in all patients using a 1.5T scanner (Intera, Philips Medical Systems, Best, Netherlands) with a 32-channel phased-array cardiac surface coil. For volumetric, functional, and feature tracking analysis a steady-state free precession cine sequence (repetition time $3.4 \mathrm{~ms}$, echo time $1.7 \mathrm{~ms}$, slice thickness $8 \mathrm{~mm}$, no interslice gap, acquisition in end-expirational breath-hold) in contiguous short axis orientation covering the entire left and right ventricle, and three long axis views (2-, 3- and 4-chamber view orientation) were obtained as previously reported $[11,14]$.

For LGE imaging, gadoterate meglumine (Dotarem ${ }^{\circledR}$, Guerbet, Villepinte, France) was administered intravenously at the dose of $0.2 \mathrm{mmol} / \mathrm{kg}$ body-weight. After $10 \mathrm{~min}$, a look-locker sequence for individual adjustment of the inversion time was performed in short axis orientation. Thereafter, an inversion-recovery gradient-echo sequence (repetition time $7.1 \mathrm{~ms}$, echo time $3.2 \mathrm{~ms}$, slice thickness $8 \mathrm{~mm}$, respiratory navigator) for evaluation of LGE was acquired in the same orientations as the cine images $[11,14]$.

\section{Image analysis}

All CMR images were anonymized before DICOM transfer to a dedicated workstation. Two experienced readers, blinded to the clinical data, evaluated all images in consensus using a dedicated software (cmr42, Circle, Calgary Canada). Cine images were analyzed for right and left ventricular end-diastolic, end-systolic volumes, and for left 
ventricular myocardial mass. Ejection fractions were calculated, correspondingly. Deformation parameters were evaluated using feature tracking.

The inversion-recovery gradient-echo sequence was evaluated for presence of LGE by both readers in consensus. Subepicardial and/or intramural LGE was defined as nonischemic, whereas subendocardial and/or transmural LGE as ischemic. An example for left ventricular LGE-imaging and of the visualization of myocardial deformation of a patient with IIM is seen in Fig. 1.

\section{Statistical analysis}

All data are reported as mean value and standard deviation. In case of normally distribution in the Shapiro-Wilk test, the Student $t$ test for normally distributed variables was used. Otherwise, Mann-Whitney-U test was performed. A $\mathrm{p}$-value $<0.05$ was regarded to be statistically significant.

\section{Results}

\section{Study population}

The study group consisted of 53 patients with histologically proven idiopathic inflammatory myopathy. In all these patients basline CMR After a mean follow-up of $234.7 \pm 79.5$ days in 47 patients, the second CMR scan could be performed. There was a loss of follow-up in 6 patients. In the study group, 10 patients had dermatomyositis, 31 had polymyositis and 6 had other subforms of IIM. Twenty-five healthy, age and gender matched volunteers underwent CMR examinations as control group. Baseline characteristics and general findings of the study and control groups are illustrated in Table 1. In the study cohort, 19 patients were hypertensive (none in control group), 8 had dyslipidemia ( 2 in control group), 2 had diabetes mellitus and 8 were smokers (both none in control group). Neither patients, nor controls had a known history of myocardial disease at baseline.

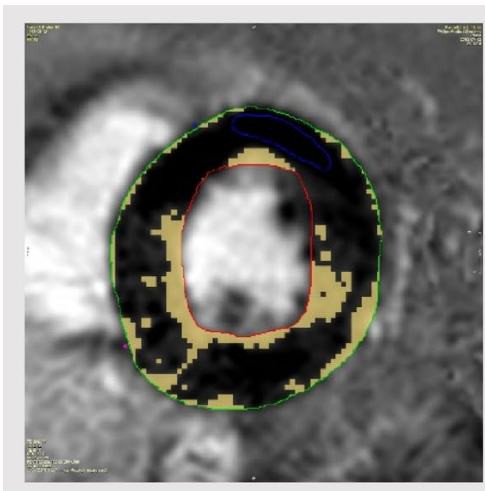

a

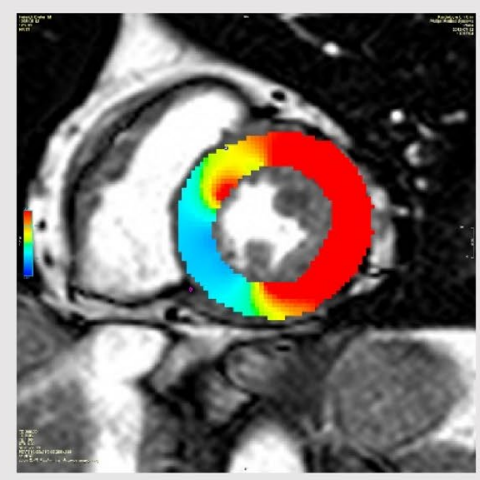

C

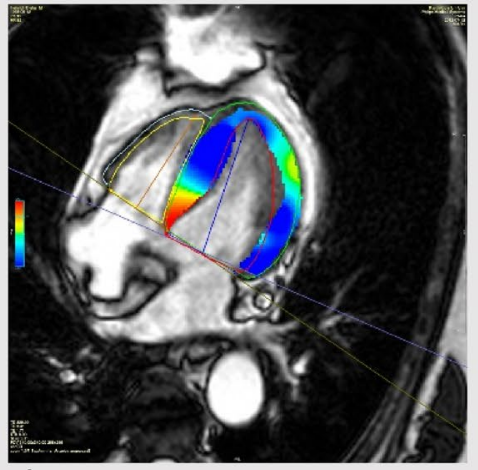

b

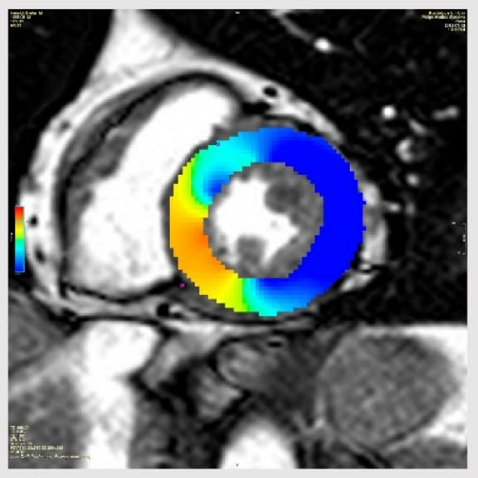

d

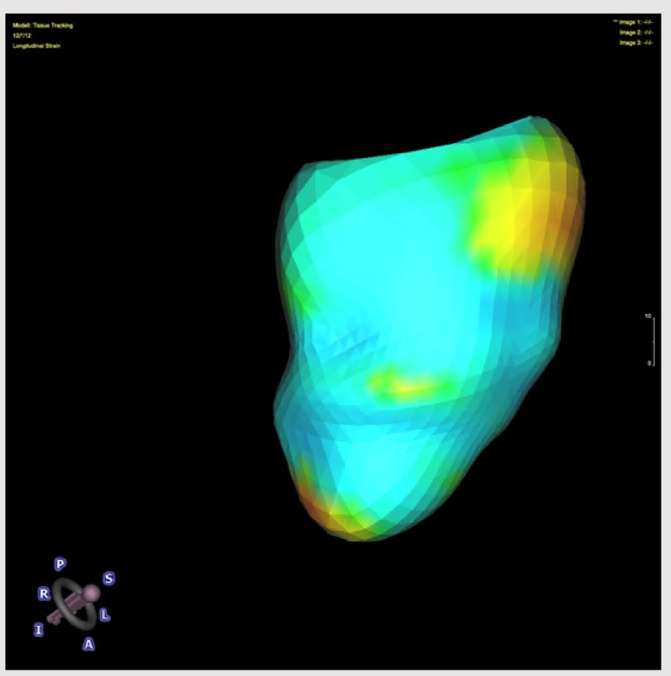

e
Fig. 1 Patient with idiopathic inflammatory myopathy. a Short axis view of a late gadolinium enhancement sequence (yellow colored parts with pathological enhancement), b long axis four-chamber view in end-systole with illustration of the longitudinal strain, $\mathbf{c}$ and d short axis view in end-systole with illustration of radial (c) and circumferential strain d, e 3D-reconstruction of the left ventricle colored according to the longitudinal strain 
Table 1 Baseline characteristics and general results of patients with Idiopathic inflammatory myopathy (IIM) and Controls

\begin{tabular}{lllr}
\hline & $\begin{array}{l}\text { Patients with IIM } \\
(\mathrm{N}=47)\end{array}$ & Control group $(\mathrm{N}=25)$ & p-value \\
\hline Age (years) & $54.3 \pm 15.4$ & $53.4 \pm 10.75$ & 0.771 \\
Males (n) & $22(46.8 \%)$ & $12(48.0 \%)$ & $0.924^{*}$ \\
BMI $\left(\mathrm{kg} / \mathrm{m}^{2}\right)$ & 26.5 & 25.1 & 0.861 \\
Hypertension (n) & $19(40.4 \%)$ & $0(0 \%)$ & $<0.001^{*}$ \\
Type 2 diabetes (n) & $8(17.0 \%)$ & $2(8 \%)$ & $0.512^{*}$ \\
Dyslipidemia (n) & $2(4.3 \%)$ & $0(0 \%)$ & $0.030^{*}$ \\
Smoker (n) & $8(17.0 \%)$ & $0(0 \%)$ & $0.030^{*}$ \\
LVEF $(\%)$ & $64.4 \pm 5.8$ & $62.0 \pm 5.6$ & 0.102 \\
LVEDVi (ml $\left./ \mathrm{m}^{2}\right)$ & $76.5 \pm 16.9$ & $70.8 \pm 11.1$ & 0.141 \\
LV mass $(\mathrm{g})$ & $90.2 \pm 28.5$ & $102.0 \pm 28.5$ & 0.102 \\
LV mass $/ \mathrm{LVEDVi}\left(\mathrm{g} / \mathrm{ml} / \mathrm{m}^{2}\right)$ & $1.195 \pm 0.31$ & $1.448 \pm 0.35$ & 0.003 \\
RVEF $(\%)$ & $63.5 \pm 5.9$ & $58.8 \pm 6.9$ & 0.003 \\
RVEDVi $\left(\mathrm{ml} / \mathrm{m}^{2}\right)$ & $66.3 \pm 15.4$ & $66.7 \pm 14.0$ & 0.923 \\
LVGRS $(\%)$ & $36.8 \pm 7.0$ & $42.4 \pm 7.6$ & 0.003 \\
LVGCS (\%) & $-18.8 \pm 2.4$ & $-20.9 \pm 2.4$ & $<0.001$ \\
LVGLS (\%) & $-17.4 \pm 2.4$ & $-20.1 \pm 2.2$ & $<0.001$ \\
\hline
\end{tabular}

*Mann-Whitney-U test used

\section{CMR results}

All CMR scans were performed without complications. Left ventricular function and volumes were normal in all patients (with LVEF $>55 \%$ considered as normal). In comparison to our healthy control group, IIM patients had similar LVEF $(64.4 \pm 5.8 \%$ vs. $62.0 \pm 5.6 \%, \mathrm{p}=0.102)$ and left and right ventricular volumes. In difference, they had a statistically reduced strain parameters, including left ventricular global radial strain (LVGRS) $(36.8 \pm 7.0 \%$ vs. $42.4 \pm 7.6 \%$, $\mathrm{p}=0.003)$, left ventricular global circumferential strain (LVGCS) $(-18.8 \pm 2.4$ vs. $-20.9 \pm 2.4, \mathrm{p}<0.001)$ and left ventricular global longitudinal strain (LVGLS) $(-17.4 \pm 2.4 \%$ vs. $-20.1 \pm 2.2 \%, \mathrm{p}<0.001)$. There was also a non-significant reduction in left ventricular mass $(90.2 \pm 28.5 \mathrm{~g}$ vs. $102.0 \pm 28.5 \mathrm{~g}, \mathrm{p}=0.102)$, which reaches significance when reported to the LVEDVi (left ventricular mass/LVEDVi) $\left(1.448 \pm 0.35 \mathrm{~g} / \mathrm{ml} / \mathrm{m}^{2}\right.$ vs. $1.195 \pm 0.31 \mathrm{~g} / \mathrm{ml} /$ $\left.\mathrm{m}^{2}, \mathrm{p}=0.003\right)$. There were significant higher scores for right ventricular ejection fraction (RVEF) in the study-group than in controls $(63.5 \pm 5.9$ vs. $58.8 \pm 6.9, \mathrm{p}=0.003)$.

A pathological LGE was found in 28 patients, while the LGE pattern was always non-ischemic. In comparison, patients without LGE had higher values for LVEF, LVGLS, right ventricular global longitudinal strain (RVGLS) and left ventricular longitudinal diastolic strain rate. There were no significant differences in other volumetric or deformation parameters (see Table 2). In one case, a new pathological LGE was found at follow up.

At follow-up, every systolic functional parameter was reduced. Whereas the relative reduction of LVEF was modest but significant $(66.6 \% \pm 6.2$ to $64.4 \% \pm 5.8$, $\mathrm{p}=0.006$ ), the decrease in deformation parameters including LVGRS, LVGCS, LVGLS and RVGLS was more substantial (see Fig. 2). There was also a reduction in left ventricular systolic longitudinal strain rate $(-1.12 / \mathrm{s} \pm 0.25$ to -1.03 $/ \mathrm{s} \pm 0.25, \mathrm{p}=0.011)$ and left ventricular diastolic longitudinal strain rate $(1.15 / \mathrm{s} \pm 0.26$ to $0.95 / \mathrm{s} \pm 0.24, \mathrm{p}<0.001)$. Left ventricular mass was reduced at time of follow-up (96.1 $\mathrm{g} \pm 31.8$ to $90.2 \mathrm{~g} \pm 28.5, \mathrm{p}=0.004)$. There was no significant difference in left or right ventricular volumes or RVEF (see Table 3).

\section{Discussion}

Cardiac events are a common finding in IIM patients and a major cause of mortality [3, 4]. Early diagnosis of cardiac involvement in inflammatory muscle diseases is therefore necessary to allow for early detection of patients at risk [5, 6]. Significantly lower myocardial deformation parameters in IIM patients in comparison to a healthy control cohort were observed in our study. Moreover, LVEF was not significantly reduced, suggesting that a change in myocardial deformation can be observed before decrease of ejection fraction. Therefore, myocardial deformation parameters may serve to detect high risk IIM patients in good time before cardiac events occur.

We used CMR feature tracking and not echo speckle tracking for the strain analysis in our trial, since CMR is the current gold standard for the evaluation of ventricular volumes, the global and regional function. This is due to 
Table 2 Volumetric and strain parameters of patients with idiopathic inflammatory myopathies (IIM), comparison between patients with and without late gadolinium enhancement (LGE)
Fig. 2 Relative reduction of left ventricular ejection fraction (LVEF), left ventricular global radial strain (LVGRS), left ventricular global circumferential strain (LVGCS), left ventricular global longitudinal strain (LVGLS) and right ventricular global longitudinal strain (RVGLS) from baseline to follow-up

\begin{tabular}{|c|c|c|c|}
\hline & IIM without LGE $(n=19)$ & IIM with LGE $(n=28)$ & p-value \\
\hline LVEF (\%) & $69.4 \pm 4.9$ & $64.8 \pm 6.4$ & 0.016 \\
\hline LVEDVi $\left(\mathrm{ml} / \mathrm{m}^{2}\right)$ & $77.5 \pm 15.9$ & $75.0 \pm 16.2$ & 0.612 \\
\hline LV mass $(\mathrm{g})$ & $106.3 \pm 36.1$ & $89.9 \pm 27.9$ & 0.095 \\
\hline $\operatorname{RVEF}(\%)$ & $65.2 \pm 6.3$ & $62.5 \pm 5.5$ & 0.134 \\
\hline RVEDVi $\left(\mathrm{ml} / \mathrm{m}^{2}\right)$ & $70.1 \pm 14.2$ & $66.5 \pm 13.2$ & 0.397 \\
\hline LVGRS (\%) & $41.3 \pm 7.1$ & $38.9 \pm 7.1$ & 0.254 \\
\hline LVGCS (\%) & $-20.0 \pm 2.0$ & $-19.7 \pm 2.5$ & 0.662 \\
\hline LVGLS (\%) & $-19.7 \pm 2.2$ & $-17.8 \pm 2.2$ & 0.005 \\
\hline RVGLS (\%) & $-31.0 \pm 2.8$ & $-28.3 \pm 4.0$ & 0.014 \\
\hline $\begin{array}{l}\text { Left ventricular systolic } \\
\text { longitudinal strain rate } \\
\text { (/sec) }\end{array}$ & $-1.161 \pm 0.24$ & $-1.093 \pm 0.26$ & 0.365 \\
\hline $\begin{array}{l}\text { Left ventricular diastolic } \\
\text { longitudinal strain rate } \\
(/ \mathrm{sec})\end{array}$ & $1.266 \pm 0.25$ & $1.063 \pm 0.24$ & 0.008 \\
\hline
\end{tabular}

Table 3 Volumetric and strain parameters in patients with idiopathic inflammatory myopathies (IIM), comparison between baseline and follow-up measurements

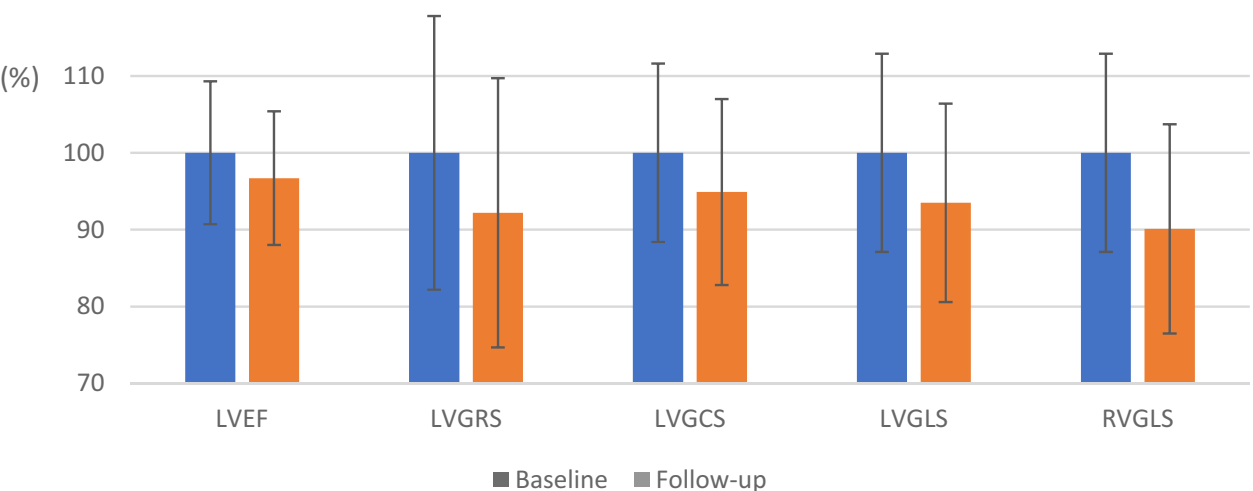

\begin{tabular}{|c|c|c|c|}
\hline & IIM at baseline $(\mathrm{N}=47)$ & IIM at follow-up $(\mathrm{N}=47)$ & p-value \\
\hline LVEF $(\%)$ & $66.6 \pm 6.2$ & $64.4 \pm 5.8$ & 0.006 \\
\hline LVEDVi in $\left(\mathrm{ml} / \mathrm{m}^{2}\right)$ & $76.0 \pm 15.9$ & $76.5 \pm 16.9$ & 0.751 \\
\hline LV mass $(\mathrm{g})$ & $96.1 \pm 31.8$ & $90.2 \pm 28.5$ & 0.004 \\
\hline RVEF (\%) & $63.5 \pm 5.9$ & $62.4 \pm 5.8$ & 0.136 \\
\hline RVEDVi (ml/m²) & $67.8 \pm 13.5$ & $66.3 \pm 15.4$ & 0.184 \\
\hline LVGRS (\%) & $39.9 \pm 7.1$ & $36.8 \pm 7.0$ & $<0.001$ \\
\hline LVGCS (\%) & $-19.8 \pm 2.3$ & $-18.8 \pm 2.4$ & $<0.001$ \\
\hline LVGLS (\%) & $-18.6 \pm 2.4$ & $-17.4 \pm 2.4$ & $<0.001$ \\
\hline RVGLS (\%) & $-29.4 \pm 3.8$ & $-26.5 \pm 4.0$ & $<0.001$ \\
\hline $\begin{array}{l}\text { Left ventricular systolic } \\
\text { longitudinal strain rate } \\
(/ \mathrm{sec})\end{array}$ & $-1.12 \pm 0.25$ & $-1.03 \pm 0.25$ & 0.011 \\
\hline $\begin{array}{l}\text { Left ventricular diastolic } \\
\text { longitudinal strain rate } \\
(/ \mathrm{sec})\end{array}$ & $1.15 \pm 0.26$ & $0.95 \pm 0.24$ & $<0.001$ \\
\hline
\end{tabular}

its ability to image $3 \mathrm{D}$ volumes unaffected by the patients habitus. For this reason, CMR has a superior inter- and intraobserver variability compared to echo. It is possible but yet not proven that CMR is thereby better than echo in the question of deformation parameters. In a current study by Pryds et al. speckle tracking echocardiography and feature tracking CMR have shown a good correlation. Both methods are thereby not interchangeable at custom, since the global 
longitudinal strain was measured higher and the global radial and circumferential strain lower with speckle tracking echocardiography [15]. Otherwise, CMR has the advantage of the possibility of a non-invasive tissue characterization with LGE and increasingly with parametric mapping. Deformation parameters seem to correlate with LGE in some entities but cannot replace it according to current knowledge [16]. At least, speckle tracking echocardiography can fail due to low image quality and requires an experienced examiner, while - of course-CMR is more expensive and not as widely available.

In other disease entities, deformation parameters have been shown to have a high diagnostic and predictive value. Doerner and colleagues found that especially in patients with preserved left ventricular function and suspected myocarditis, left ventricular strain parameters in combination with the Lake-Louise-Criteria improve diagnostic performance. Patients with myocarditis had a significantly reduced LVGLS in comparison to healthy volunteers $(-17.2 \pm 4.9 \%$ vs. $-13.3 \pm 6.2 \%, p<0.001)$ [17]. We showed a quite similar difference in longitudinal strain between IIM patients and healthy counterparts.

Another recent trial showed that LVGLS was the only independent predictor of functional recovery in patients with myocarditis [18]. A high predictive value of LVGLS was also shown for other myocardial diseases like Takotsubo cardiomyopathy, ST-segment elevation myocardial infarction and chemotherapy induced cardiomyopathy $[12,13,19]$. Future investigations are warranted to evaluate the relationship between early changes in deformation parameters and clinical outcome in IIM patients.

For no obvious reason we had a difference in RVEF favoring the IIM-study group. We cannot explain whether this is due to an unknown confounder or small sample size. In fact, the values were not pathological [20] and there were no clinical signs of right ventricular insufficiency neither in the IIM nor in the control group.

Another potentially important diagnostic parameter for different cardiomyopathies is presence of LGE, which is a marker of fibrosis and consequently of irreversible myocardial remodeling. It is a common finding, inter alia, in infectious myocarditis [8]. As previously shown, a pathological LGE pattern is also often present in IIM patients [11]. In our cohort, LGE presence was associated with reduction in LVEF, LVGLS and RVGLS. Lee et al. have shown a correlation between LGE and occurrence of cardiac events in patients with acute myocarditis [21]. Such relationships are also conceivable for IIM patients and should be subject of further investigation.

In our study cohort, a significant decrease of LVEF and all strain parameters were observed between baseline and followup. A recent meta-analysis could exclude a potential correlation between age and deformation parameters [22]. Hence, a subclinical deterioration of myocardial function over time can be excluded. Observed worsening of functional parameters in our study Is consequently due to the underlying disease. Thus, a single CMR examination may not provide enough information to detect patients at risk. A follow-up CMR exam can provide information about functional worsening and thereby contribute to therapeutic decision as therapy escalation.

\section{Study limitations}

The main limitation of this study is the small sample size. This is essentially due to the fact that IIM are rare diseases according to the Rare Diseases Act of 2002 of the United States. In fact, our study population is large compared to others found in literature. Another limitation is the unicentric study design. Our data should be validated in larger cohorts of multicentric studies. Furthermore, our study was not designed to examine the impact of imaging findings on clinical decision-making and patient outcome. Whether a change in immunosuppressive therapy because of MR-findings has an influence on clinical outcome should be investigated in a prospective randomized trial.

\section{Conclusions}

Cardiac involvement in IIM was frequently identified. We have shown that CMR including LGE and feature tracking provides additional information to classical ejection fraction measurements and may improve diagnostic work-up of IIM patients in future.

Funding Open Access funding enabled and organized by Projekt DEAL. The trial was partly funded by a research Grant of Guerbet.

Data availability The datasets used and/or analyzed during the current study are available from the corresponding author on reasonable request.

\section{Compliance with ethical standards}

Conflict of interest The authors declares that they have no conflict of interest.

Ethical approval The study was approved by the ethics committee of the University of Ulm (Approval no. 13/10). Every participant gave written informed consent.

Informed consent Every participant gave written informed consent.

Open Access This article is licensed under a Creative Commons Attribution 4.0 International License, which permits use, sharing, adaptation, distribution and reproduction in any medium or format, as long as you give appropriate credit to the original author(s) and the source, provide a link to the Creative Commons licence, and indicate if changes were made. The images or other third party material in this article are 
included in the article's Creative Commons licence, unless indicated otherwise in a credit line to the material. If material is not included in the article's Creative Commons licence and your intended use is not permitted by statutory regulation or exceeds the permitted use, you will need to obtain permission directly from the copyright holder. To view a copy of this licence, visit http://creativecommons.org/licenses/by/4.0/.

\section{References}

1. Ernste FC, Reed AM (2013) Idiopathic inflammatory myopathies: current trends in pathogenesis, clinical features, and up-to-date treatment recommendations. Mayo Clin Proc 88(1):83-105. https ://doi.org/10.1016/j.mayocp.2012.10.017

2. Longo DL, Dalakas MC (2015) Inflammatory muscle diseases. N Engl J Med 372(18):1734-1747. https://doi.org/10.1056/NEJMr a1402225

3. Zhang L, Wang G, Ma L et al (2012) Cardiac involvement in adult polymyositis or dermatomyositis: a systematic review. Clin Cardiol 35(11):686-691. https://doi.org/10.1002/clc.22026

4. Diederichsen LP (2017) Cardiovascular involvement in myositis. Curr Opin Rheumatol 29(6):598-603. https://doi.org/10.1097/ BOR.0000000000000442

5. Allanore Y, Vignaux O, Arnaud L et al (2006) Effects of corticosteroids and immunosuppressors on idiopathic inflammatory myopathy related myocarditis evaluated by magnetic resonance imaging. Ann Rheum Dis 65(2):249-252. https://doi.org/10.1136/ $\operatorname{ard} .2005 .038679$

6. Caforio ALP, Adler Y, Agostini C et al (2017) Diagnosis and management of myocardial involvement in systemic immune-mediated diseases: a position statement of the European Society of Cardiology Working Group on myocardial and pericardial disease. Eur Heart J 38(35):2649-2662. https://doi.org/10.1093/eurheartj/ ehx321

7. Schmid J, Liesinger L, Birner-Gruenberger R et al (2018) Elevated cardiac troponin $\mathrm{T}$ in patients with skeletal myopathies. $\mathrm{J}$ Am Coll Cardiol 71(14):1540-1549. https://doi.org/10.1016/j. jacc.2018.01.070

8. Friedrich MG, Sechtem U, Schulz-Menger J et al (2009) Cardiovascular magnetic resonance in myocarditis: a JACC white paper. J Am Coll Cardiol 53(17):1475-1487. https://doi.org/10.1016/j. jacc.2009.02.007

9. Ponfick M, Gdynia H, Kassubek J et al (2011) Cardiac involvement in juvenile overlap-myositis detected by cardiac magnetic resonance imaging. Int J Cardiol 152(1):e25-e26. https://doi. org/10.1016/j.ijcard.2010.10.001

10. Huber AT, Bravetti M, Lamy J et al (2018) Non-invasive differentiation of idiopathic inflammatory myopathy with cardiac involvement from acute viral myocarditis using cardiovascular magnetic resonance imaging T1 and T2 mapping. J Cardiovasc Magn Reson 20(1):11.https://doi.org/10.1186/s12968-018-0430-6

11. Rosenbohm A, Buckert D, Gerischer N et al (2015) Early diagnosis of cardiac involvement in idiopathic inflammatory myopathy by cardiac magnetic resonance tomography. J Neurol 262(4):949956. https://doi.org/10.1007/s00415-014-7623-1
12. Stiermaier T, Lange T, Chiribiri A et al (2018) Left ventricular myocardial deformation in Takotsubo syndrome: a cardiovascular magnetic resonance myocardial feature tracking study. Eur Radiol. https://doi.org/10.1007/s00330-018-5475-2

13. Gavara J, Rodriguez-Palomares JF, Valente F et al (2018) Prognostic value of strain by tissue tracking cardiac magnetic resonance after ST-segment elevation myocardial infarction. JACC Cardiovasc Imaging 11(10):1448-1457. https://doi.org/10.1016/j. jcmg.2017.09.017

14. Buckert D, Dewes $P$, Walcher $T$ et al (2013) Intermediate-term prognostic value of reversible perfusion deficit diagnosed by adenosine CMR: a prospective follow-up study in a consecutive patient population. JACC Cardiovasc Imaging 6(1):56-63. https ://doi.org/10.1016/j.jcmg.2012.08.011

15. Pryds K, Larsen AH, Hansen MS et al (2019) Myocardial strain assessed by feature tracking cardiac magnetic resonance in patients with a variety of cardiovascular diseases - a comparison with echocardiography. Sci Rep 9(1):11296. https://doi. org/10.1038/s41598-019-47775-4

16. Erley J, Genovese D, Tapaskar N et al (2019) Echocardiography and cardiovascular magnetic resonance based evaluation of myocardial strain and relationship with late gadolinium enhancement. J Cardiovasc Magn Reson 21(1):46. https://doi.org/10.1186/s1296 8-019-0559-y

17. Doerner J, Bunck AC, Michels G et al (2018) Incremental value of cardiovascular magnetic resonance feature tracking derived atrial and ventricular strain parameters in a comprehensive approach for the diagnosis of acute myocarditis. Eur J Radiol 104:120-128. https://doi.org/10.1016/j.ejrad.2018.05.012

18. Luetkens JA, Petry P, Kuetting D et al (2018) Links- und rechtsventrikulärer Strain im Krankheitsverlauf der akuten Myokarditis: eine kardiale MRT Studie (left and right ventricular strain in the course of acute myocarditis: a cardiovascular magnetic resonance study). Rofo 190(8):722-732. https://doi. org/10.1055/a-0585-0271

19. Negishi K, Negishi T, Hare JL et al (2013) Independent and incremental value of deformation indices for prediction of trastuzumabinduced cardiotoxicity. J Am Soc Echocardiogr 26(5):493-498. https://doi.org/10.1016/j.echo.2013.02.008

20. Kawel-Boehm N, Maceira A, Valsangiacomo-Buechel ER et al (2015) Normal values for cardiovascular magnetic resonance in adults and children. J Cardiovasc Magn Reson 17:29. https://doi. org/10.1186/s12968-015-0111-7

21. Lee JW, Jeong YJ, Lee G et al (2017) Predictive value of cardiac magnetic resonance imaging-derived myocardial strain for poor outcomes in patients with acute myocarditis. Korean J Radiol 18(4):643-654. https://doi.org/10.3348/kjr.2017.18.4.643

22. Vo HQ, Marwick TH, Negishi K (2018) MRI-derived myocardial strain measures in normal subjects. JACC Cardiovasc Imaging 11(2 Pt 1):196-205. https://doi.org/10.1016/j.jcmg.2016.12.025

Publisher's Note Springer Nature remains neutral with regard to jurisdictional claims in published maps and institutional affiliations. 